\section{Six million patients wait as politicians argue over the elective recovery plan}

\author{
Chris Ham
}

Plans to tackle the backlog of routine care in the NHS in England, due to be published today, have been delayed because of disagreement within the government over the weekend. Reports indicate that disagreement centred on the Treasury's concerns about the targets for reducing the backlog and more significantly on the reluctance of the Chancellor of the Exchequer to provide the prime minister with ammunition to bolster his credibility after Sue Gray's report into the partygate affair. ${ }^{1}$ Patients have been left in limbo until these differences can be resolved.

The delayed plan was originally intended to be published in November 2021. The resurgence of covid in the wake of the omicron variant prevented this happening and only now, with covid pressures reducing, has NHS England felt the time is right to prepare a public launch. The plan, prepared under the leadership of Jim Mackey, chief executive of Northumbria Healthcare NHS Foundation Trust, is expected to set out how the NHS will transform the delivery of care, expand physical capacity, give priority to patients in greatest need, and offer patients more information, choice, and control over their own care.

The last of these aims will be enabled by the launch of the "My Planned Care" online platform which, over time, is intended to provide information on waiting times and support for patients to maintain or achieve their fitness to ensure their surgery can go ahead. Other priorities include expanding community diagnostic centres to provide more convenient options for people to get important tests and scans, making outpatient care more flexible by giving patients access to assessments and appointments at home and to arrange follow ups when convenient, and further separating elective and emergency work.

The NHS has often been described as a political football-usually when one or other of the main political parties has claimed to be its true defender. What is unusual about this week's events is the way in which divisions within the governing party have led to the NHS being weaponised. There could be no clearer indication of tensions at the heart of government and the febrile atmosphere within Downing Street. While it is likely that a way forward will be found and the plan published shortly, the causes of the delay are not going away any time soon.

The proximate causes relate to the fragility of the prime minister's position ahead of publication of Sue Gray's full report and the conclusion of the Metropolitan Police's investigations into parties in Downing Street. The more fundamental causes concern anxiety in government about surveys showing rising public pessimism about the NHS, particularly with a general election due to be held in 2024 at the latest. ${ }^{2}$

Planned increases in national insurance contributions to fund the new health and social care levy, the proceeds of which will help fund the elective recovery plan, are another factor. With views in the governing party on the principle and timing of the levy hardening, the pressure to demonstrate that the NHS is using additional funding effectively is likely to grow. This, as well as the high politics at work, helps explain the Treasury's eleventh hour intervention on the plan.

It would be wrong to interpret this week's events as a storm that will pass when other issues take over. The public and political salience of the NHS mean that its performance will be under constant scrutiny for the foreseeable future with its leaders and staff held to account for delivering the commitments set out in the plan. It will be important that these commitments are both ambitious and realistic, with enough latitude for clinicians to continue treating patients on the basis of clinical need and priority.

The challenge will be to reduce the number of people waiting longer than 104 weeks and then 52 weeks for treatment, while also giving priority to the reduction of cancer waiting times and treating other patients with urgent and potentially life threatening conditions. The NHS demonstrated its ability to innovate at speed during the pandemic response and has shown what can be done on elective recovery when the demands of covid have permitted. Chronic staffing shortages continue to be the main constraint, placing a premium on clinical teams being supported to find more effective ways of delivering care to as many patients as possible.

The six million people in England currently on waiting lists are a reminder of what is at stake. Each person is coping with a mixture of anxiety, uncertainty, and pain. Politicians would do well to remember this as they resolve their differences. The public will not easily forgive them at the next general election if they fail.

Chris Ham was chief executive of The King's Fund from 2010 to 18

Competing interests: none declared

Provenance and peer review: commissioned, not peer reviewed

Rishi Sunak is loyal to me, insists Boris Johnson as pair unite over NHS backlog. The Times. https://www.thetimes.co.uk/article/2a4afde8-879311ec-8600-c48a9935f856?shareToken $=\mathrm{d} 2 \mathrm{~d} 32139127501617849 \mathrm{~cd} 369 \mathrm{e} 8 \mathrm{~d} 38 \mathrm{f7}$

2 Public perceptions of the NHS and social care: performance, policy and expectations - The Health Foundation https://www.health.org.uk/publications/long-reads/public-perceptions-performance-policy-and-expectations 